\title{
Experimental-analytical method of technological processes modeling in education
}

\author{
German I. Efremov ${ }^{1}$ and Julia A. Geller ${ }^{1, *}$ \\ ${ }^{1}$ Moscow Technological Institute, 199334, Moscow, Russia
}

\begin{abstract}
The article considers general modeling techniques used in the study in education at different stages. The classification of different types of models and main stages of the simulation are considered. It is shown that in the course "Process of simulation" for technical areas of the Universities required the category of "Experimental-analytical simulation method". For example, a new textbook for bachelors "Modeling of chemical-technological processes" shows that the section facilitates the compilation of process models in general; gives the possibility of studying the process at different levels; describes the nonlinear properties of the simulation objects, and to obtain refined adjustment of the model according to the experiment. The use of models of high accuracy can improve the quality of education.
\end{abstract}

\section{Introduction}

It is widely known the statement of academician V. A. Izvozchikov about information (computerized) society, when "in all spheres of life and activity of the members of the society are included the computer, telematics, Informatics other means as instruments of intellectual work that enables great speed to make calculations and to process any information, to simulate actual and foreseeable events, processes, phenomena, control production, to automate training, etc." [1]. According to some experts, the U.S. will complete the overall transition to the information society by the year 2020, Japan and most countries of Western Europe and probably Russia - to 2030-2040 years.

As we move towards the information society the big changes are taking place in the education. One of the fundamental problems facing modern education, - to make it more accessible and more effective for each person. This accessibility has economic, social, and technological aspects. The technological aspects include the ability to effectively simulate large volumes of databases, to implement effective, precise mathematical models of different processes.

The study of the course "Informatics", which includes section modeling, is implemented in the framework of continuing education students. In school this course under different name variants studied from 2 to 11 classes. So, in high school exists for studying the course "Informatics and ICT". In the Universities also is studied the course "Computer science" [2], and in the content of these courses deals with the problems of different types of modeling.

In the school curriculum "Informatics and ICT" is one of the important category "Technology of information modeling", which deals with the issues of modeling dependencies between different variables, mathematical models (for dependencies between variables represented in a mathematical form), tabular and graphical models, statistical models (is obtained by analysing of statistical data), regression of the mathematical model (obtained including the method of "least squares"), the model correlations (on the basis of correlation analysis), optimum planning models. There are also processes for optimal information modeling in the determination of planned values of indicators at given limited resources and the subjects to the achievement of predetermined goals.

Undergraduate course "Information technology" is included in mathematical and natural-science cycle of disciplines working curricula for all engineering specialties. For example, the textbook N. In. Makarova and V. B. Volkov [2]. In this tutorial - the Basics of the simulation are given in-depth knowledge on the specifics of modeling (conceptual, physical, structural-functional, simulation, computer), discusses in detail the parameters of models (universality, accuracy, adequacy, efficiency, visibility, limb, computability, modularity, algorithmsuite, simplification).

\section{Classification of models}

In the textbook for high schools [2] the classification of models is considered. For the purpose of application the model are classified into cognitive (characterized as accurately as possible), pragmatic (a means of organizing practical action) and instrumental (a means to build, study and use of pragmatic and cognitive models). For the levels of simulation the models are classified into empirical (constructed on the basis of empirically

*Corresponding author: y geller@mti.edu.ru 
established dependencies between input and output parameters), the theoretical (on the basis of the mathematically described dependencies) and semiempirical (based on approximations of the empirical relationships using mathematical functions to meet the challenges of modeling accuracy).

There's also the classification of the models on the hierarchical levels of the simulation models of microlevel (showing objects or processes of the lowest, indivisible in parts of the level), macro-level (display the features or processes of middle or senior managers in a hierarchical structure) and models of meta-level (displays processes or objects that interact with the prototype model macro-level). The purpose of modeling at the meta-level - a more accurate reproduction of the environment (input parameters) models of the macrolevel. The classification of the models according to the nature of the relationship to the external environment: an open model (continuous energy- and material exchange with the environment) and closed model (has a weak relationship with the external environment, or does it not matter).

Models are classified according to the method of presentation of the object's properties: analytic (completely determined via a set of mathematical functions), algorithmic (described by algorithm or complex of algorithms determining the functioning and its development) and simulation (plotted for the test, study the object by variation of some or all of the model parameters).

The name "simulation" model exists, because it allows to simulate the behavior of real complex systems without detailed description of the internal mechanism of this behavior. Mathematical simulation model as a complex system is represented as a conjunction of individual elements, some of which can be described analytically (using functional dependencies), and others constitute "black boxes" whose functions are approximable by probabilistic dependencies. It should be noted that the simulation models can be not only mathematical, they can also be implemented in other ways, including using models or by gaming simulation. In the classification models for causation can be allocated to models deterministic (uniquely identify the set of output parameters for each used combination of input parameters) and non-deterministic, or stokhasticheskaya, i.e. probabilistic models (assuming the probabilistic nature of input parameters as probabilistic nature of the functions or the algorithms used for their processing).

Classification of models in relation to the process time includes the dynamic model, where explicitly uses time as an input parameter, and the static model, in which the time parameter explicitly among the input parameters is not present. Static models are usually used to find the boundary or the optimal values of certain parameters. Usually the dynamic model can be implemented in time with some zoom (deceleration or acceleration).

Pattern classification by applications driven not only by the characteristics of the models themselves, since the principles of modeling remain the same regardless of the scope of application of the model, as the specificity of the collection and preparation of source material for simulation and specific features of the description of the subject area (scientific, technical, social, military, economic).

In practice, the widely used pattern classification methodology for applications: training models (created to support the educational process and normally partially reproduce the functionality of the modeled object or its parts that it is impossible to observe and study in your facility's operation), games (model in the form of a game in different situations reproduces the processes occurring in a complex system, they are often developed for training of skills and abilities and can be built both spontaneously and in an organized manner).

This classification includes the experimental model (constructed to reproduce the properties of an artificial object and study it under different conditions, and in some cases it can be even harder and more expensive than object modeling) and simulation model (used to simulate the behavior of the object or simulation of running processes in complex systems).

Classification of models according to the method of presentation includes: a physical model (due to its physical structure, shape, energy performance, they reproduce the simulated object, and it is characterized by immediate, tangible, and not information form, the reproduction of certain features of the prototype) and information model (where the engine model is the information, and models can be formalized, for example, a mental model or abstract painting and formalized, when they are embodied in the form of symbols, sayings, pictures or drawings which value is specified). The formal model can be as computer and non-computer. It is important to emphasize the difference between material and information models.

Media - paper, on which you are drawing, or the computer that simulates the operation of a complex mechanism is always material. However, the means of modeling the information model is intangible.

Given the diversity of ways of model classifying shows that the modeling world is rich and diverse. It should be noted that it is often difficult to relate the model to one group. For example, a computer game is simultaneously mathematical, and geometric, and gaming, and digital (with a certain level of performance), and analog, discrete, and continuous, and algorithmic, and simulation model.

\section{Main stages of modelling}

It is important to consider the main stages of computer modeling and simulation. The essence of modeling is to simulate the phenomenon. The accuracy of the simulation is determined by comparing obtained from the modeling result, with the object of study, and to assess their degree of similarity. In accordance with the main stages of computer modeling include: formulation of the problem (formulated in general form, in the form of verbal descriptions, sufficient to fulfill the purpose of 
modeling), the development of models, computer experiment and the analysis of simulation results.

Developing a model involves a thorough study of the model and the description obtained in the study results in a formal computer language, highlights the model elements, connections between them, it is significant for the research of characteristics and parameters. The final stage is the obtaining of information model of the object. The information model can be represented in the form of data tables, the set of mathematical relationships, charts and graphs, logical statements and other formal descriptions.

Computer experiment depends on what type of simulation was chosen in accordance with the purpose of simulation; it can wears both short and long-term in nature, and its results can be used directly after receiving or they will serve as a basis to further refine and adjust the model.

The analysis of simulation results is to establish the adequacy of the model the object of research, i.e. to determine the degree of similarity of the model with its original object. The successful result of the evaluation of the product model shows a sufficient level of knowledge of object, of the correctness of the principles underlying the modeling, the suitability of the chosen algorithm, that is, that the used model works. This model can be used for further research of an object under new conditions, in which the real object has not yet been studied.

Often the first results of the simulation do not satisfy these requirements. This means that at one of the stages there are errors and inaccuracies. This requires more research and a corresponding change to a computer program simulation. There is also a class of objects for which the various reasons has not developed analytical model is either not developed methods for their solution. The complexity of the processes taking place in "nonideal" systems often makes it impossible to formalize. In this case, when it is necessary to study such complex systems, a simulation model is built. Experimentation with such a model is called simulation.

\section{Simulation}

The essence of simulation modeling [2] is as follows:

1. The system is split into a large number of functional units (decomposition).

2. Each block is replaced by the model "black box" with inputs and outputs and the function of dependence of output parameters from the input.

3. As a conversion function can be an analytical expression, probability function or approximation function on the basis of experimental data.

4. Models of functional blocks are combined into a model system that simulates the behavior of real complex systems.

The basis of simulation modeling are the processes of decomposition, determine the function of each block, which converts the input parameters in the input, and then building a model based on synthesis system model functional blocks.
All simulation models are model type "black box". This means that they provide for the output of the input signal of the system if its interactive subsystem receives an input signal. Therefore, to obtain the necessary information or results, you must implement the "run" simulation models, rather than "solve" them. Simulation models are not capable of forming a solution in the form which it occurs in the analytical models, and can serve only as a tool for the analysis of the system behavior in conditions that are determined by the experimenter.

The researcher must consider the feasibility of using simulation modeling in the presence of any of the following conditions:

- Formalization of the model impossible, or yet to be developed analytical methods for solving the formulated mathematical models. This category includes many models of mass service.

- Analytical methods are available but the mathematical procedures are so complex and time-consuming that the simulation allows to get a simpler solution.

- Analytical solutions exist, but their implementation is impossible due to insufficient mathematical training to existing staff. In this case, you should weigh the costs of designing, testing and work on the simulation model with the costs associated with the invitation of experts from outside.

- In addition to the evaluation of certain parameters, it is desirable to implement in the simulation model for the monitoring process over a certain period.

- The difficulty of setting up experiments and observations in a real environment; a relevant example is the study of the behavior of spacecraft in the conditions of interplanetary flight.

- For long-term operation of the systems or processes may need to compress the timeline. Simulation modeling allows you to fully control the timing of the process under study, because the phenomenon can be slowed or accelerated as desired. This category includes, for example, the problem of studying the dynamics of development of human settlements at various levels.

Modern simulation depending on the purpose of modeling and solving tasks involves one of three approaches to modeling: system dynamics (considers the system as a set of variables, interconnected feedback loops); discrete-event simulation (describes a system as a hierarchical sequence of elements and operations receive, capture, unloading, registering, placing, issuing) and agent-based modeling (describes a system as a set of complex objects -the agents associated with certain rules, reveals their behavior).

In the textbooks of different levels are also considered a software simulation environment. Analysis of the market of information technologies allows to identify the main trends in modern modeling systems [2]. The dominant basic concepts of formalization and structuring in modern modelling systems for discrete simulation are used: a system based on process descriptions (process description) or to network concepts (network paradigms), such as Extend, Arena, ProModel, Witness, Taylor, Gpss/H-Proof. For systems intended for continuous simulation are used models and methods of system dynamics such as Powersim, Vensim, Dynamo, 
Stella, Ithink etc. And it exists the powerful systems to expand their functionality supported alternative to the concept of formalization. For example, in system Powersim, Ithink the integrated apparatus of discrete modeling and, Vice versa, Extend, ProcessModel supports continuous simulation.

In many bachelor and specialist program exists a course on "Process simulation" [3], the content of which is adjusted depending on a direction of study. This course is included in professional cycle curriculum. In this course, typically have sections:

- Types of modeling: physical and mathematical. The connection between the physical and mathematical modeling. The advantages and disadvantages of types of modeling. The analogy of the processes in the original object and in the model. The classification models. Approximate and exact models. The mathematical model of "elementary" processes.

- Similarity theory, its application in the modeling. Geometric, kinetic and dynamic similarity. Similarity criteria. Theorems of similarity. The differential equations for transfer of momentum, heat and mass. Application of the theory of similarity in physical modeling. The determination of the conditions of similarity. The analogy of the simulation of the transfer of momentum, heat and mass.

- Mathematical modeling. A mathematical description. The equation balances. Methods of creating mathematical models. The method of "black box". Classification of mathematical models.

- Computational simulation. Criteria-based models for stationary transfer. Models of continuous media and pseudo-homogeneous models. The diffusion model. The model in disperse media. Modeling of transients.

- Method of experiment planning. Passive and active experiment. The planning matrix. A fractional factorial experiment.

- The method of determining the reaction of the object on a standard perturbations: a single impulse, step, sinusoidal.

- Comparison of methods for constructing mathematical models. Assesses the adequacy and model identification. The Pearson Criterion. The choice of the mathematical model.

- Modeling of reactor processes. Reactor perfect mixing and perfect displacement. A mathematical model of a catalytic reactor. The choice of reactor. The stability of reactor circuits and industrial reactors.

- Optimization of chemical processes. The optimality criterion and the requirements for it. Geometric interpretation of objective function. Global and local Optima. Analytical methods of optimization. Dynamic, linear and nonlinear programming. Gradient-based methods. A method of relaxation. The method of steepest descent. Scan method. Simplex method. Method of deductions. Comparison of different optimization techniques.

Feature a new tutorial "Modeling of chemicaltechnological processes" for bachelors [3] is the presence of the section "Experimental-analytical simulation method". From our point of view this section is necessary for all Universities, because it allows to obtain models of high accuracy. When this method is used the theory of process based on the physical nature of the phenomena occurring in the facility-adjusted model the results of the experiment. In this case, decomposition of complex phenomena into simple, elementary processes, which are easier to investigate. Model complexity is determined, as a rule, the complexity of the investigated object and the degree of accuracy required of the calculation results. After analyzing the influence of process steps, unimportant factors are discarded and only those that are most significant. Mathematical description is made in the form according to the main process. The influence of other elementary processes taken into account by using coefficients as the main dependence.

Advantages of the method: the separation of a complex process into individual stages and the study of the process in parts, which facilitates the compilation of process models in general; the possibility of studying the process at different levels; description of nonlinear properties of object modeling and refined to adjust the model according to the experiment.

Disadvantages of the method: the difficulty of finding reliable theory for complex processes, the impossibility of its use with an unknown mechanism of the process; found the model coefficients change depending on the conditions of the experiments, so experimental and analytical model of the fair is often only in a certain range, which made the experiment.

When comparing a purely empirical methods and experimental - analytical methods of construction of mathematical models it should be noted that experimentally - analytical method has the advantage over purely experimental method in that it reflects both the theory of the process and unaccounted for in the model of the phenomena, by introducing effective factors. In the first method the experiment required for obtaining the model itself and the second - to determine the values specifying its coefficients. The same method can be used in the creation of fundamentally new technological processes.

There are the most complete characteristic of hygroscopic materials and its built for the full range of parameter changes. The importance of determining mathematical models of isotherms is that on their basis it is possible to find the equilibrium concentrations for the processes of sorption and desorption, which in turn are included in the kinetic model of drying, wetting, soaking, rinsing, etc. As an example of application of this method in the textbook [3] discusses mathematical models of isotherms of sorption-desorption (the dependence of the equilibrium moisture content of the material $\mathrm{w}$ on the concentration of absorbed substances.

Considered models for the three (main) types of isotherm BET classification (by author's name: Brunauer, Emmett and Teller). Other types of isotherms are derived and differ only in the shape of the hysteresis loop. It should be noted that the second type isotherm (Sshaped) can be obtained by summation of the first (convex) and the third (concave) types of isotherms. The models are built for the sorption of benzene vapor at a temperature of about 20 degree Celsius with different structure of pores. The drying process of different 
materials occurs most rapidly at elevated temperatures. So it is more convenient scaling of isotherms taken at relatively low temperatures, at higher, using a temperature dependence. The effect of temperature on the position of the isotherm described by the dependence in the form of the equation of Arrhenius. To illustrate the temperature dependence of the constructed model isotherms of desorption of moisture from the corn seed at six temperatures. On the basis of the results obtained we have constructed the politherms, i.e. isotherms for different temperatures in spatial Cartesian coordinates. It should be noted that politherms, compared to the conventional isotherms are of greater visibility to the impact of factors than the simple isotherms constructed at different temperatures in a planar coordinate system. The correlation coefficient for the models polyterm amounted of 0.991 , which indicates a high accuracy of the models.

The models of diffusion processes for external and internal tasks of the processes are also proposed. Modeling of the drying process is carried out on the basis of the solution of the diffusion equation with boundary condition in the form of a flux, with subsequent adjustment of the coefficients. The mathematical model for diffusion of substances from the material into an infinite half-space slab with zero initial condition with the subsequent application of the Laplace transform is analyzed. The modified quasi-stationary method (MQM) applied for simulation of diffusion processes is also considered. The developed a modified quasi-stationary method [3] is innovative. It allows to obtain with high accuracy mathematical model for the internal tasks of the process. The model is derived based on modified quasi-stationary is a dimensionless and generalize equation of the kinetics of diffusion processes for internal tasks. For the external problem of the drying process the two - and three-parameter models are proposed.

These models tested on the experimental data of drying of asbestos and cotton fabric. The correlation coefficient is 0,9998 .

For the modification by the quasistationary method is used, two parameters - the characteristic time of the process and the hydrodynamic factor are introduced. The model of filtration drying of the layer of granules of ammonium phosphate $45 \mathrm{~mm}$ thickness is obtained (correlation coefficient amounted to 0.9991). Another example of application of equations MQM for modeling the kinetics of drying of disperse polymer material with shrinkage and convective drying of pellets of polycaproamide (PCA) at a temperature of $110 \mathrm{oC}$ are the work of the authors $[4,5]$. In this simulation, the Pearson correlation coefficient amounted to is 0.998 , i.e. the obtained models for different drying temperatures, describe well the experimental data. Similar calculations and graphs were made for drying the pellets of polyethylene terephthalate. Based on the proposed methods the generalized model of kinetics of internal and external challenges of the process are obtained.

In another paper of the author [6] the optimal mathematical model for the cascade of ideal mixing reactors, which are isothermal first order reaction, it is obtained, based on the method of dynamic programming. It was found, that the optimal values of the residence time for all reactors are equal, respectively, the ratio of concentrations in the reactor and volumes of all cascade reactors are also equal.

The considered above high precision models help to improve the quality of education at different stages.

\section{Conclusion}

1. The general modeling techniques used in the study of this issue in education at its various stages are considered.

2. The classification of different types of models and main stages of the simulation is considered.

3. It is shown, that in the course "Process simulation" for technical areas of the University it is required the category of "Experimental-analytical simulation method".

4. For example, in a new textbook for bachelors "Modeling of chemical-technological processes" it is shown that the section facilitates the compilation of process models in general; gives the possibility of studying the process at different levels; describe the nonlinear properties of the simulation object, and to obtain refined adjustment of the model according to the experiment.

5. The use of models of high accuracy can improve the quality of education at different stages.

\section{References}

1. I.G. Semakin, E.K. Henner, Computers and ICT, The textbook for 10-11 class (Moscow, 2013)

2. N.I. Makarova, V. B. Volkov, Computer Science. Textbook for high schools (Peter, 2011)

3. G.I. Efremov Modeling of chemical processes, Textbook for high schools (INFRA-M, 2016)

4. Y.A. Geller, G. I. Efremov, Modelling the kinetics of drying of pellets of polymers. In X International conference Educational environment today and tomorrow, p. 242-243, (2015)

5. Y.A. Geller, G.I. Efremov Drying of dispersed materials based on their shrinkage. In $\mathrm{X}$ International conference Educational environment today and tomorrow, p. 244-245 (2015)

6. G.I. Efremov, Optimization of parameters of the cascade of ideal mixing reactors. In X International conference Educational environment today and tomorrow, p. 289-290 (2015) 